\title{
Spin diffusion at finite electric and magnetic fields
}

\author{
Y. Qi and S. Zhang \\ Department of Physics and Astronomy, University of Missouri-Columbia, Columbia, MO 65211
}

(Dated: October 24, 2018)

\begin{abstract}
Spin transport properties at finite electric and magnetic fields are studied by using the generalized semiclassical Boltzmann equation. It is found that the spin diffusion equation for non-equilibrium spin density and spin currents involves a number of length scales that explicitly depend on the electric and magnetic fields. The set of macroscopic equations can be used to address a broad range of the spin transport problems in magnetic multilayers as well as in semiconductor heterostructure. A specific example of spin injection into semiconductors at arbitrary electric and magnetic fields is illustrated.

PACS numbers: 72.25.Dc, 72.25.-b
\end{abstract}

Spin transport phenomena have been identified in various layered systems including magnetic multilayers, magnetic tunnel junctions, and semiconductor heterostructure. The partial list of these phenomena includes the spin injectiondetection in metals [1], giant magnetoresistance [2], enhanced Gilbert damping [3, 4], spin angular momentum transfer [5, 6] and spin current induced spin excitation [7], ferromagnetic resonant [8], tunnel magnetoresistance [9], and spin Hall effect in semiconductors 10, 11. Most recently, there are emerging interests in the spin injection and detection in semiconductor spintronics $[12$. Several theoretical models based on a macroscopic drift-diffusion equation for each spin channel has been proposed 13, 14, 15].

The central observation of all these interesting spin-dependent transport phenomena is the key role played by the electron spin. Unlike the physics of strongly correlated systems, most of the spin transport phenomena can be understood in terms of the semiclassical description of electrons in the diffusive scattering limit as long as the spin degree of freedom is properly being taken care of quantum mechanically. In this letter, we construct a set of generalized spin dependent macroscopic equations from the spinor form of the Boltzmann equation. We have found that these macroscopic equations can self-consistently determine the charge density, spin density, charge current and spin currents. The above mentioned spin dependent phenomena can be naturally understood by solving the macroscopic equations with various experimental boundary conditions. In particular, one can predict spin transport in semiconductor heterostructure at arbitrarily strengths of electric and magnetic fields.

Let us start with the spinor form of the semiclassical Boltzmann equation that describes the equation of motion for spin-half conduction electrons in an external electric field $\mathbf{E}$ and an effective magnetic field $\mathbf{H}_{e}$

$$
\frac{\partial \hat{F}}{\partial t}+\mathbf{v} \cdot \nabla_{\mathbf{r}} \hat{F}+\frac{e}{m^{*}}\left(\mathbf{E}+\mathbf{v} \times \mathbf{H}_{e}\right) \cdot \nabla_{\mathbf{v}} \hat{F}-\frac{i}{\hbar}[\hat{H}, \hat{F}]=-\left(\frac{\partial \hat{F}}{\partial t}\right)_{\text {colli }}
$$

where $\hat{F}$ is the distribution function that depends on the position $\mathbf{r}$, momentum $\mathbf{p}$ (or velocity $\mathbf{v}=\mathbf{p} / m^{*}$ ), and time $t, \mathbf{H}_{e}$ is the effective magnetic field, $\hat{H}$ is the spin dependent Hamiltonian which can be written in spin space as $\hat{H}=-\mu_{B} \boldsymbol{\sigma} \cdot \mathbf{H}_{e}$. The right-hand side of the equation is the collision term. Although the validity of the semiclassical Boltzmann equation is limited to the wave packet picture of the electron motion, the quantum mechanical nature of the spin has been explicitly taken into account by the spinor form of the distribution function and the spin dynamics is included in the commutator $[\hat{H}, \hat{F}]$ for the spin variable.

Since we are interested in spin-dependence of the transport, we will make a few simplifications to Eq. (1). First, the effect of the magnetic field on the orbital motion is discarded, i.e., we have set $\left(\mathbf{v} \times \mathbf{H}_{e}\right) \cdot \nabla_{\mathbf{v}} \hat{F}=0$; noticeably, this orbital term is in fact needed if one is interested in the ferromagnetic resonance under an applied AC and DC magnetic fields [8]. Second, we take the collision term in a simplest s-wave form by using momentum-independent relaxation time approximations for both non-spin-flip and spin-flip scattering, i.e.,

$$
\left(\frac{\partial \hat{F}}{\partial t}\right)_{\text {colli }}=\frac{\hat{F}-\bar{F}}{\tau}+\frac{2}{\tau_{s f}}\left[\bar{F}-\frac{\hat{I}}{2} \operatorname{Tr} \bar{F}\right]
$$

where $\tau$ and $\tau_{s f}$ are the momentum and spin relaxation times, $\bar{F} \equiv(1 / 4 \pi) \int d \Omega_{\mathbf{k}} \hat{F}(\mathbf{r}, \mathbf{v})$ is the angular average over the $\mathbf{k}$-space, $\hat{I}$ is the $2 \times 2$ unit matrix in spin space, and $T r$ represents the trace over spin space. In ferromagnetic materials, the momentum relaxation time $\tau$ is also spin-dependent; this can be easily generalized by using a $2 \times 2$ matrix for $\tau$. For the notation simplicity, we write it as a number. Finally, we should consider only the layered structure so that the spatial dependence of the distribution is limited to one-dimension; this is a practical approximation since the 
three-dimensional inhomogeneity will lead to a complicated spatial-dependence of the current density that makes the calculation of the conductance extremely tedious. Let us denote the direction perpendicular to the layered structure as $x$-direction.

With above simplifications, Eq. (1) becomes

$$
\frac{\partial \hat{F}}{\partial t}+v_{x} \frac{\partial \hat{F}}{\partial x}+\frac{e E_{x}}{m^{*}} \frac{\partial \hat{F}}{\partial v_{x}}-\frac{\mu_{B}}{\hbar} \boldsymbol{\sigma} \cdot\left(\mathbf{H}_{e} \times \mathbf{f}\right)=\frac{\hat{F}-\bar{F}}{\tau}+\frac{2 \bar{F}-\hat{I} \operatorname{Tr} \bar{F}}{\tau_{s f}}
$$

where we have introduced a spin-dependent distribution vector $\mathbf{f}$ by separating the distribution function into spin dependent and spin independent parts, $\hat{F} \equiv f_{0} \hat{I}+\boldsymbol{\sigma} \cdot \mathbf{f}$, so that the commutator $(1 / i \hbar)[\hat{H}, \hat{F}]=\left(\mu_{B} / \hbar\right) \boldsymbol{\sigma} \cdot\left(\mathbf{H}_{e} \times \mathbf{f}\right)$.

Equation (3) is known as the generalized Bloch equation [16]. One of major tasks in the spin transport theory is to solve this integro-differential equation with desired precision for various physical structures. Here, we do not intend to solve exactly the distribution function as a function of the momentum, position and time for a detail structure. Rather we construct a set of macroscopic equations from the above equation by integrating out the momentum variable so that the spatial dependence of the macroscopic spin dependent transport coefficients can be established for a wide range of interesting physical systems.

The first set of the macroscopic equations are obtained by directly summing over the momentum (velocity). By separately writing down the spin dependent and spin independent parts of Eq. (3), we have

$$
\frac{\partial n_{0}(x, t)}{\partial t}+\frac{\partial j_{0}(x, t)}{\partial x}=0
$$

and

$$
\frac{\partial \mathbf{m}(x, t)}{\partial t}+\frac{\partial \mathbf{j}_{m}(x, t)}{\partial x}-\frac{2 \mu_{B}}{\hbar} \mathbf{H}_{e} \times \mathbf{m}(x, t)=-\frac{\mathbf{m}(x, t)}{\tau_{s f}}
$$

where we have defined the charge density $n_{0}(x, t)=\sum_{\mathbf{v}} \operatorname{Tr} \hat{F}$, the spin density $\mathbf{m}(x, t)=\sum_{\mathbf{v}} \operatorname{Tr}(\boldsymbol{\sigma} \hat{F})$, the charge current density $j_{0}(x, t)=\sum_{\mathbf{v}} \operatorname{Tr}\left(v_{x} \hat{F}\right)$, and the spin current density $\mathbf{j}_{m}(x, t)=\sum_{\mathbf{v}} \operatorname{Tr}\left(\boldsymbol{\sigma} v_{x} \hat{F}\right)$. The above equations are exact macroscopic equations for charge and spin: Eq. (4) is the law of charge conservation, and Eq.(5) governs the motion of spin in the presence of spin relaxation due to spin flip scattering and spin precession due to a magnetic field. To determine four macroscopic quantities $\left(n_{0}, j_{0}, \mathbf{m}\right.$ and $\left.\mathbf{j}_{m}\right)$ we need to construct two more equations; this can be furnished by timing $v_{x}$ at the both sides of Eq. (3) and summing over v. Again, after separating the spin dependent and spin independent parts of Eq. (3), we find

$$
\frac{\partial j_{0}(x, t)}{\partial t}+\overline{v_{x}^{2}} \frac{\partial n_{0}(x, t)}{\partial x}+\frac{e E_{x}}{m^{*}} n_{0}(x, t)=-\frac{j_{0}(x, t)}{\tau}
$$

and

$$
\frac{\partial \mathbf{j}_{m}(x, t)}{\partial t}+\overline{v_{x}^{2}} \frac{\partial \mathbf{m}(x, t)}{\partial x}+\frac{e E_{x}}{m^{*}} \mathbf{m}(x, t)-\frac{2 \mu_{B}}{\hbar} \mathbf{H}_{e} \times \mathbf{j}_{m}(x, t)=-\frac{\mathbf{j}_{m}(x, t)}{\tau}
$$

In deriving the above closed form, we have used the "mean field" approximation $\sum_{\mathbf{v}} v_{x}^{2} \frac{\partial \hat{F}}{\partial x} \approx \overline{v_{x}^{2}} \sum_{\mathbf{v}} \frac{\partial \hat{F}}{\partial x}$. In another words, we only consider the first two polynomials (zeroth and first orders) of the distribution function in the expansion respect to the direction of the momentum. This approximation has been used for most of diffusion theories and it is valid as long as the system is not too much anisotropic. In principle, however, we should keep higher orders of polynomials and one requires to use more macroscopic variables such as $\mathbf{Q}_{n}=\sum_{\mathbf{v}} v_{x}^{n} \operatorname{Tr}(\boldsymbol{\sigma} \hat{F})$ where $n=0$ is for the spin (number) density, $n=1$ is for the spin current density, $n=2$ is for the spin energy density, and so forth. Truncating the expansion at $n=2$ makes the equations for the macroscopic variables in the closed form, but it sacrifices the accuracy in the solution of the Bloch equation for anisotropic systems. However, we argue that inclusion of higher orders does not in fact improve much of the accuracy of the calculation since we have already made an approximation in deriving the Bloch equation by assuming a $\mathbf{k}$ independent relaxation time.

Equations (4) and (6) describe the non-equilibrium charge density and charge current density. Alone with the Poisson equation, Eqs.(4) and (6) completely determine the charge carrier distribution in a layered structure in the presence of arbitrary electric fields. A well-known example is the semiconductor p-n junction where the electric field in the depletion region is quite large, thus the charge diffusion should be calculated by keeping all orders of the electric field in solving Eqs. (4) and (6). Here our focus is on the spin part of the macroscopic equations, Eqs. (5) and (7).

The spin diffusion equation can be readily derived by expressing the spin current density in terms of the spin density from Eq. (7) and by inserting the resulting expression into Eq. (5). Let us first consider the time independent case. As 
Eq. (7) contains a cross product of the spin current density vector and the magnetic field, it is convenient to separate the components parallel and perpendicular to the magnetic field, i.e.,

$$
j_{m}^{z}=-\tau \overline{v_{x}^{2}} \frac{\partial m_{z}}{\partial x}-\frac{e E_{x} \tau}{m^{*}} m_{z}
$$

and

$$
j_{m}^{ \pm}=-\frac{1}{1 \mp 2 i \hbar^{-1} \mu_{B} \tau H_{e}}\left(\tau \overline{v_{x}^{2}} \frac{\partial m_{ \pm}}{\partial x}+\frac{e E_{x} \tau}{m^{*}} m_{ \pm}\right)
$$

where we have assumed a uniform magnetic field in the z-direction, and defined the perpendicular components of the spin current density $j_{m}^{ \pm}=j_{m}^{x} \pm i j_{m}^{y}$ and the spin density $m_{ \pm}=m_{x} \pm i m_{y}$. By placing them into Eq. (5), we arrive at the spin diffusion equations for the longitudinal and transverse spin density,

$$
\frac{\partial^{2} m_{z}}{\partial x^{2}}+\frac{\operatorname{Sign}\left(E_{x}\right)}{\lambda_{E}} \frac{\partial m_{z}}{\partial x}+\left(\frac{1}{\lambda_{E} \lambda_{c}}-\frac{1}{\lambda_{s d f}^{2}}\right) m_{z}=0
$$

and

$$
\frac{\partial^{2} m_{ \pm}}{\partial x^{2}}+\frac{\operatorname{Sign}\left(E_{x}\right)}{\lambda_{E}} \frac{\partial m_{ \pm}}{\partial x}+\left(\frac{1}{\lambda_{E} \lambda_{c}}+\frac{1}{\lambda_{h}^{2}}-\frac{1}{\lambda_{s d f}^{2}} \mp \frac{i}{\lambda_{s}^{2}}\right) m_{ \pm}=0
$$

where $\operatorname{Sign}\left(E_{x}\right)=1(-1)$ for $E_{x}>(<) 0$. We have introduced a number of relevant lengths: the usual spin-flip scattering length $\lambda_{s d f}=\sqrt{\overline{v_{x}^{2}} \tau \tau_{s f}}$, the length associated with acceleration of electrons by the electric field $\lambda_{E}=$ $m^{*} \overline{v_{x}^{2}} / e\left|E_{x}\right|$, the length due to non-uniform electric field (or due to charge accumulation in the depletion layers) $\lambda_{c}^{-1}=\left|\left(1 / E_{x}\right) d E_{x} / d x\right|$, the spin precession length induced by the magnetic field $\lambda_{h}=\hbar \sqrt{\overline{v_{x}^{2}}} / 2 \mu_{B} H_{e}$, and the precessional spin density decay length $\lambda_{s}=\sqrt{\hbar \tau \overline{v_{x}^{2}} / 2 \mu_{B} H_{e}}$.

The spin diffusive equations, Eq. (10) for the longitudinal spin density and Eq. (11) for the transverse spin density, are our central results. For the longitudinal spin density, the spin diffusion is not affected by the magnetic field since we have disregarded the electron orbit motion. The electric field as well as its spatial derivative have significant effects on the spin diffusion for semiconductor heterostructure where the interface regions are expected to possess large space charges and thus large electric fields. For example, for a non-degenerate semiconductor, $m^{*} \overline{v_{x}^{2}} \approx k_{B} T$, and thus $\lambda_{E}$ is only about $100 \AA$ for a typical electric field of $2.5 \times 10^{4}(\mathrm{~V} / \mathrm{cm})$ at $\mathrm{T}=300 \mathrm{~K}$. In a moderated doped interface, the gradient of the electric field in the depletion layer is large so that $\lambda_{c}$ is also of the order of $100 \AA$. Such small $\lambda_{E}$ and $\lambda_{c}$ make the diffusion length highly anisotropic: the spin diffusion is much easier when the direction of the diffusion is same as the direction of the electric field; otherwise, the spin diffusion is prohibited. For a metallic layer, however, the electric field effect is small since $\lambda_{E}$ is typically of the order of $10 \mu m$ if we take $m^{*} \overline{v_{x}^{2}} \approx 1$ (eV) (a fraction of the Fermi energy) and $E_{x}$ is of the order of $10^{3}(\mathrm{~V} / \mathrm{cm})$ for the extremely high current density. Thus, the commonly used spin diffusion equation which neglects the electric field is justified for metallic multilayers.

We point out that a similar spin diffusion equation for the longitudinal spin density has recently derived from the spin-dependent drift-diffusion equations by Yu and Flatté [13], and Albrecht and Smith [14] with no magnetic fields. Our derivation from the Boltzmann equation generalizes their results significantly. First, we are able to address the longitudinal and transverse spin diffusion at any magnetic field; the spintronics application is inevitably involving the magnetic field. Second, we include the additional length scale $\lambda_{c}$ due to non-uniformity of electric fields at the depletion layers; this effect has been neglected all together in the previous treatment. Third, we have started from the semiclassical Boltzmann equation so that we have a controlled access on the approximations made in deriving the spin diffusion equations; the deviation of the macroscopic equations from the exact solutions of the Boltzmann equation can be compared in various cases [17.

We now turn to a discussion on the transverse spin density and spin current density. The spin diffusion equations involve two more length scales associated with the magnetic field in addition to the length scales in the longitudinal diffusion equation. The total spin current density decaying length would depend on the detail magnitudes of these lengths scales. The magnetic field enters in two places: one is to compete with the spin flip scattering (notice the positive sign of $1 / \lambda_{h}^{2}$ ) and the other is a precessional decay of spin density due to scattering and precession (notice the imaginary $i$ ). To give out a quick estimation of the magnetic field on the transverse spin diffusion, we take an example of the non-degenerate semiconductor again. We find that the magnetic field of the order of 1000 (Oe) can make $\lambda_{h}$ and $\lambda_{s}$ comparable to $\lambda_{s d f}$. Therefore, one must consider the spin diffusion by including both finite electric and magnetic fields in a realistic spintronics device. 
Our generalized spin diffusion equations in the presence of finite electric and magnetic fields can be used to a broad range of spin transport phenomena. By taking the limiting cases, many previously known spin diffusion phenomena are recovered. The giant magnetoresistance for currents perpendicular to the plane of the layers is a simplest example where both electric and magnetic fields are assumed small [1]. The phenomenon of the spin angular momentum transfer with non-collinear magnetization vectors of two ferromagnetic layers can be studied by taking the limit of a large internal magnetic field and a vanishingly small electric field [18]. The ferromagnetic resonant experiments can be analyzed via the reduction of the diffusion constant due to a large magnetic field [8]. One can also derive a solution of the time-dependence of the spin accumulation in magnetic multilayers by keeping the time dependence diffusion term in Eq. (11).

Finally, we illustrate an important application of our spin diffusion equation in a ferromagnetic/tunnel barrier/semiconductor heterostructure. To make our calculation much simpler, we assume a uniform electric field near the interface of the insulator barrier and the semiconductor; a detailed calculation on the self-consistently determined non-uniform electric fields due to depletion potentials and the external bias will be published elsewhere. In the present case, the solutions of the longitudinal and the transverse spin density, Eqs. (10) and (11), are simple exponential functions with the exponential decaying factors given by the above mentioned length scales. To determine absolute values of the spin density and spin polarization of the current, we need to use boundary conditions across the insulator barrier. Let us introduce a spin-dependent tunnel resistance across the barrier as $\hat{R}=R_{0}\left(\hat{I}+P \boldsymbol{\sigma} \cdot \mathbf{M}_{F}\right)$ where $\mathbf{M}_{F}$ is the unit vector in the direction of the ferromagnetic magnetization and $P$ is the tunneling polarization. The conditions for continuous of spin density and spin current density at the interface between the semiconductor and the insulator barrier completely determine the prefactors of the exponential solutions.

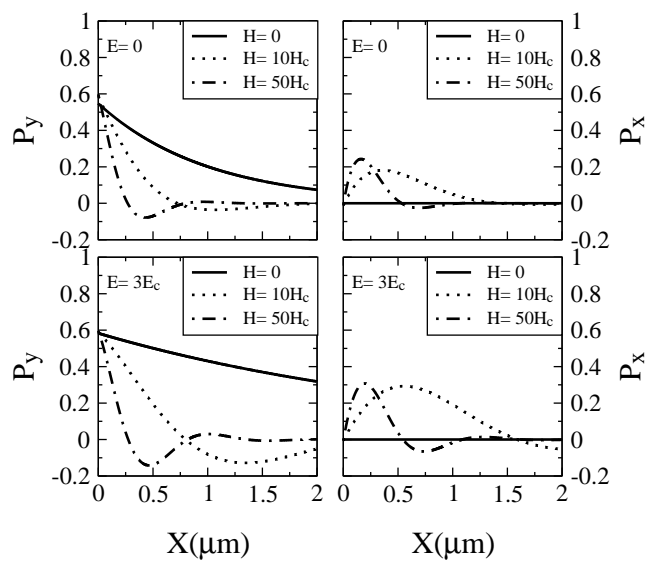

Fig.1.

Fig. 1: Spin-polarization of the current as a function of position in the semiconductor for different electric and magnetic fields. The magnetic field is applied at z-axis and the ferromagnet is polarized at $y$ direction for all panels in the figure. $P_{x} \equiv j_{\mathrm{m}}^{x} / j_{0}$ and $P_{y} \equiv j_{\mathrm{m}}^{y} / j_{0}$ are $x$ and $y$ components of the spin polarization of the current, respectively. The parameters are: the spin-diffusion length of the semiconductor is $\lambda_{s d f}=1.0 \mu \mathrm{m}$, the critical electric field and magnetic field are defined as $E_{c} \equiv k_{B} T / e \lambda_{s d f}$ and $H_{c}=\hbar / 2 \mu_{B} \tau_{s d}$, the resistance ratio $\rho_{s e m i} \lambda_{s d f} / R_{0}=0.1$ (where $\rho_{s e m i}$ is the resistivity of the semiconductor), and the polarization of the barrier resistance is $P=0.6$.

In Fig. 1, we show the non-equilibrium spin polarization of the current density as a function of the position in 
the semiconductor in different electric field and magnetic fields. Here we assume $\mathbf{M}_{F}$ is at the $y$ direction and the magnetic field is applied at the z-direction. At zero magnetic field, the electric field can enhance or reduce the spin density, depending on the direction of the field, as pointed out in [13, 14. The magnetic field has dramatic effects on the spin density and the spin polarization; the spin diffusion length is shorten significantly even for a moderate magnetic field, say, 1000 (Oe). On the other hand, one also develops a spin current in $x$-direction which was absent without the magnetic field. This $x$-component of the spin density and spin current comes from the rotation of the injected spin (originally at $y$ direction) by the magnetic field.

In summary, we have presented a set of macroscopic equations for charge density, spin density, charge current and spin current at finite electric and magnetic fields. These equations are the generalization of previous useful spin diffusions at various limiting cases. These equations can be applied to a wide range of the diffusive spin transport phenomena. This work is supported by National Science Foundation (DMR-0076171).

[1] M. Johnson and R. H. Silsbee, Phys. Rev. B35 4959 (1987).

[2] P. Holody, W. C. Chiang, R. Loloee, J. Bass, W. P. Pratt Jr., and P. A. Schroeder, Phys. Rev. B. 58, 12230 (1988).

[3] R. Urban, G. Woltersdorf, and B. Heinrich, Phys. Rev. Lett. 87, 217204 (2001).

[4] Y. Tserkovnyak, A. Brataas, and G. E. W. Bauer, Phys. Rev. Lett. 88, 117601(2002).

[5] L. Berger, Phys. Rev. B 54, 9359 (1996); J. Slonczewski, J. Magn. Magn. Mater. 159, L1 (1996).

[6] E. B. Myers, D. C.Ralph, J. A. Katine, R. N. Louie, and R. A. Buhrman, Science 285, 867 (1999).

[7] M. Tsoi, A. G. M. Jansen, J. Bass, W.-C. Chiang, M. Seck, V. Tsoi, and P. Wyder, Phys. Rev. Lett. 80, 4281(1998).

[8] G. D. Gaspari, Phys. Rev. 151, 215 (1966).

[9] J. S. Moodera, L. R. Kinder, T. M. Wong, and R. Meservey, Phys. Rev. Lett. 74, 3273 (1995).

[10] J. E. Hirsch, Phys. Rev. Lett., 93, 1834 (1999).

[11] S. Zhang, Phys. Rev. Lett., 95, 393 (2000).

[12] S. A. Wolf, D. D. Awschalom, R. A. Buhrman, J. M. Daughton, S. von Molnar, M. L. Roukes, A. Y. Chtchelkanova, and D. M. Treger, Science 294, 1488 (2001).

[13] Z. G. Yu and M. E. Flatté, cond-mat/0207471 (2002).

[14] J. D. Albrecht and D. L. Smith, Phys. Rev. B66, 113303 (2002).

[15] I. Zutić, J. Fabian, and S. Das Sarma, Phys. Rev. Lett. 88, 066603 (2002).

[16] D. C. Langreth and J. W. Wilkins, Phys. Rev. 6, 3189 (1972).

[17] Y. Qi and S. Zhang, to be published.

[18] S. Zhang, P. M. Levy, and A. Fert, Phys. Rev. Lett. 88, 236601 (2002). 